\title{
Interference and visual masking
}

\author{
Bernt Christian Skottun \\ Ullevaalsalleen $4 \mathrm{C}$ \\ 0852 Oslo \\ Norway \\ e-mail: berntchrskottun@gmail,com
}

Short title: Interference

\begin{abstract}
It is well known that a masking stimulus may reduce the visibility of a target stimulus. This has most commonly been attributed the mechanisms in the visual system. It has, however, become evident that a masking stimulus may reduce the stimulus power of a target. Such reductions which are in the stimuli have been explored in relation to spatial stimuli (Skottun, 2017, DOI: 10.3758/s13428-017-0978-3; Skottun, 2018. DOI: 10.1016/j.bbr.2018.04.016). An important aspect of visual masking is the effect of the relative temporal onset of the two stimuli (i.e., the Stimulus Onset Asynchrony, SOA). The present study sought to explore, using numerical analyses, how interference across time may manifest itself. By assuming some degree of temporal inaccuracy and a limited extent of temporal integration it was found that, depending on conditions, interference effects may take on the characteristics of either Type-A or Type-B masking. In connection with visual masking it is (tacitly) assumed that the target stimulus has the same stimulus power when presented in combination with a masking stimulus presented as when presented alone. It has earlier been shown that this cannot be assumed in connection with spatial stimuli. The present analyses make clear that this may also apply to stimuli presented at somewhat different times.
\end{abstract}

Key words: Temporal; spatial frequency; amplitude; phase; Fourier Transform; filter; interference; metacontrast. 


\section{INTRODUCTION}

It seems clear that there may be interference between visual stimuli or between different elements in visual stimuli (Skottun, 2017a, 2018a, 2018b). Thus, the introduction of a masking stimulus has the potential to reduce the stimulus power of a target stimulus. This effect takes place in the stimuli. By that is meant that it does not depend on the visual system or on the stimuli being seen. The possibility of interference has implications for visual masking in which one stimulus, the masking stimulus, reduces the visibility of another stimulus, the target stimulus. Interference between visual stimuli has been examined mainly in regard to space (Skottun, 2017a, 2018b). In visual masking the stimuli are often presented at slightly different times and the relative timing of the onset of the two stimuli (i.e. their Stimulus Onset Asynchrony, SOA) is an important variable (see, e.g., Breitmeyer, 1984). It seems therefore to be of some relevance to explore how interference may express itself across time.

In visual masking it is common to distinguish two general kinds of temporal relationships (Breitmeyer \& Ganz, 1976): (1) The largest masking effect occurs when the target and mask are presented at the same time. That is to say, it is largest when the Stimulus Onset Asynchrony (SOA) is zero and diminishes in both the forward and backward directions. This is refereed to as Type-A masking. Or, (2) the largest effect may be found when the masking stimulus is presented some time after the target. This is referred to as Type-B masking. The two types of masking are illustrated in Fig. 1 in which are re-plotted data from Fig. 3 of Hellige et al. (1979).

The task facing subjects in visual masking experiments is typically linked to the target stimulus in some manner. In these experiments it has generally been assumed, usually tacitly, that the target stimulus presented together with a masking stimulus has the same stimulus power as when present alone, and consequently that any interaction effects are the results of mechanisms in the visual system. The question of whether or not, or to what extent, there may be interactions in the stimuli has rarely, if ever, been brought up. As pointed out above, there is evidence for interference effects in the stimuli (Skottun, 2017a, 2018a, 2018b). 


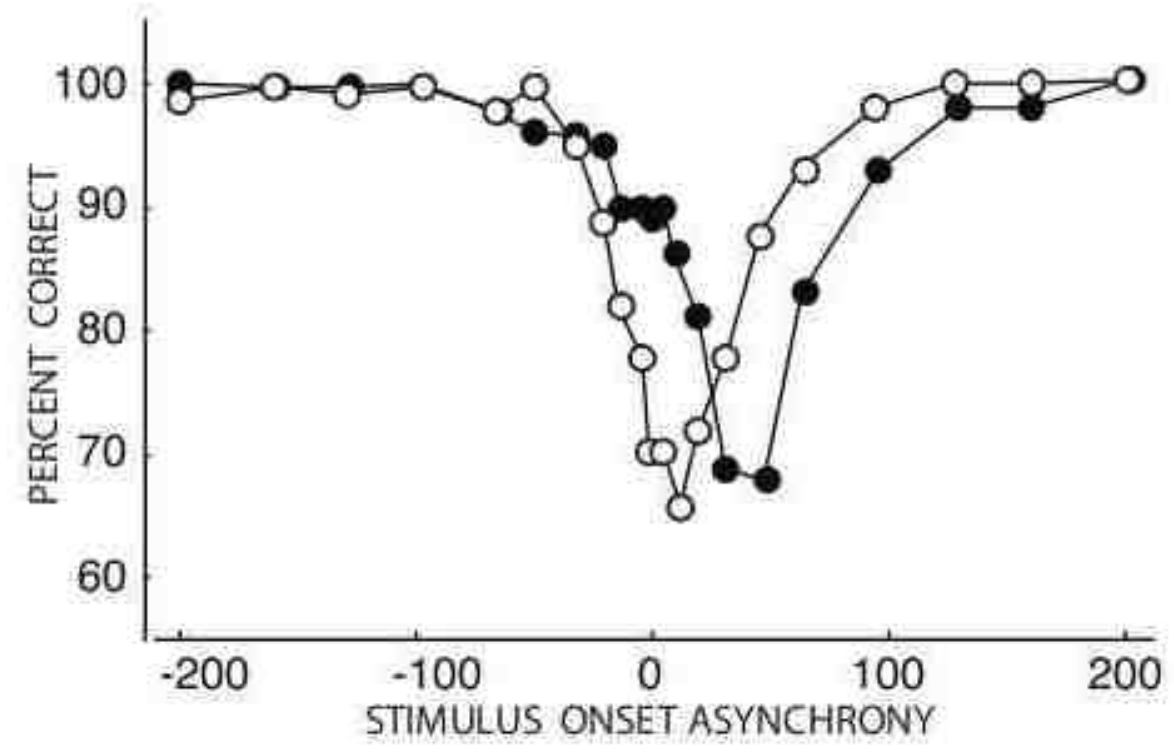

Fig. 1

Fig. 1. Masking data re-plotted from Fig. 3 of Hellige et al. (1979) showing percent correct response as a function of Stimulus Onset Asynchrony (SOA). Reductions from $100 \%$ correct reflect masking. The two functions show data for when mask and target were similar (filled symbols) and when they were different (open symbols). Note that in the case of different stimuli the masking function resembles Type-A masking (although maximum masking takes place at a small positive SOA) whereas in the case of similar stimuli the masking function displays the characteristics of Type-B masking with the maximum masking at positive SOA values, i.e. under the conditions of backward masking.

That adding stimulation may reduce the stimulus power in a target stimulus is illustrated in Fg. 2. In Fig. 2A is shown the case where a target stimulus (solid line) is both preceded and followed by masking stimuli of the same intensity (dashed lines). This renders the target stimulus invisible. In Fig. 2B is shown the case where the masking stimulus is presented only after the target stimulus. In Figs. $2 \mathrm{C}$ and $2 \mathrm{D}$ are shown cases where masking stimuli presented after the target are limited in time, and in Fig. 2E a masking stimulus of the same duration as the target is presented at some time after it. This illustrates that the case where the masking stimuli fully obscures the target (Fig. 2A) and the case where a masking stimulus is limited in time and is presented at a time distinctly different from that of the target (Fig. 2E) constitute points on a continuum. In the case shown in Fig. $2 \mathrm{~A}$ it would be most commonly assumed that the reason for why the target cannot be detected is to be found in the stimuli whereas in the case in Fig. 2E, which is equivalent to a regular masking experiment, explanations for the effect of the masking stimulus upon the target are typically sought in the visual system. Consequently, masking is commonly promoted as a means 
to explore the workings of the visual system. (Thus for instance, Enns \& Di Lollo (2000, p. 345) wrote: "Masking is a widely used and powerful way of studying visual processes", and Breitmeyer and Ogmen (2000, p. 1572) wrote "Visual backward masking ... has found increasing application as a powerful methodological tool in studies of visual information processing and as a useful instrument for investigating visual function ...

".) The present analyses take the view that also in the case depicted in Fig. 2E does one need to investigate to what extent the visibility of the target can be understood in terms of the stimuli.

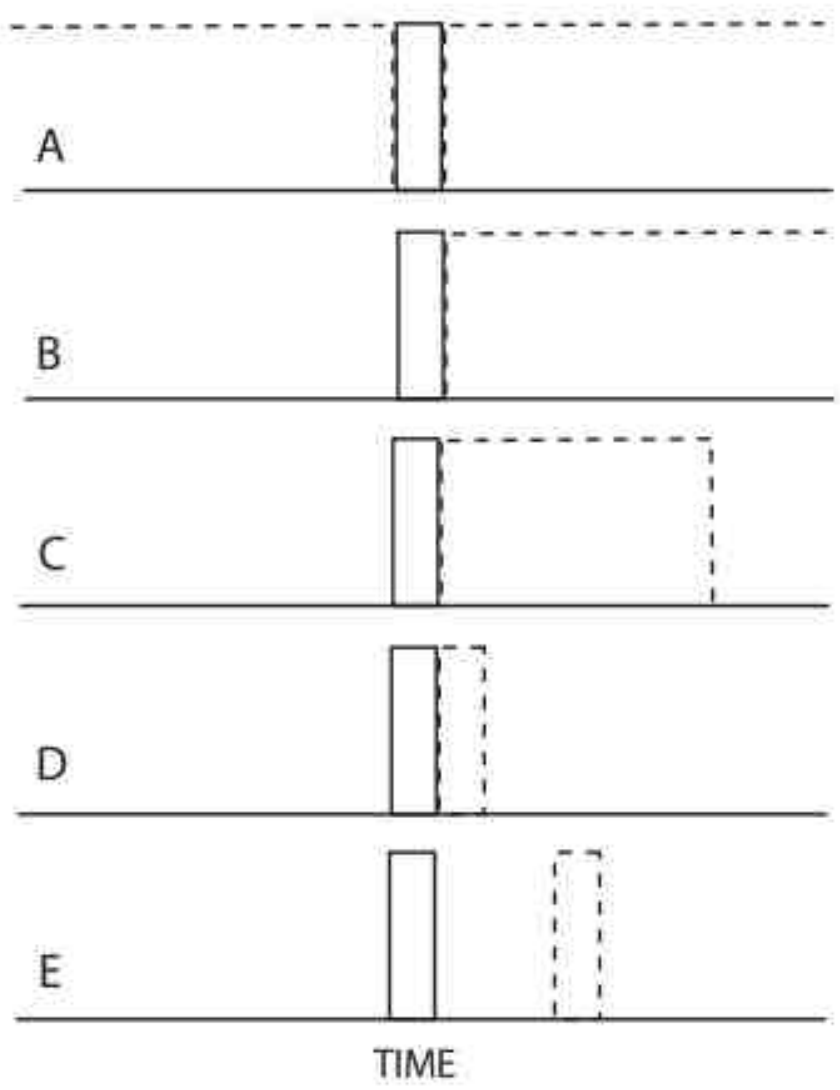

\section{Fig. 2}

Fig. 2. Temporal profiles of different configurations of target stimuli (solid lines) and masking stimuli (dashed lines). The two stimuli are depicted as having the same intensity. (A) The case where the target stimulus is both preceded and followed by masking stimuli. (B) The target is followed by a masking stimulus. (C \& D) The masking stimulus is presented after the target and is limited in duration. (E) The masking stimulus has a duration equal to that of the target and is presented at some time after the target stimulus. This last scenario amounts to a case of conventional backward masking. Thus, a target "embedded" in masking stimuli (Panel A) and a conventional masking paradigm (Panel E) represent points on a continuum. 
Interference may occur when two functions are added. This can be understood very simply in the case where the two functions are sinusoids. If the two functions are in-phase (phase difference $=2 n \pi$, where $n$ is an integer, including 0) the amplitudes will add. This is referred to as "constructive interference". On the other hand, if the functions are out-of-phase (phase difference $=m \pi$, where $m$ is an odd integer, not including 0 ) the peaks in one wave function will coincide with the troughs in the other and the amplitudes will subtract. This is referred to as "destructive interference" (for an illustration see Fig. 1 of Skottun, 2017a). Various degrees of destructive interference will also occur for phase differences between these extremes. (See also Skottun, 2017a.)

Although interference is most commonly considered in regard to spatial patterns it may also occur between patterns that vary in time. In connection with visual perception this prompts the question of whether or not there can be interference between stimuli that are presented at somewhat different points in time. Such interactions would be of some interest in relation to visual masking since masking experiments have uncovered interactions between stimuli presented at different times.

As already mentioned, explanations for the various aspects of visual masking have been sought in terms of mechanisms in the visual system. It seems, however, that before speculating on possible physiological mechanisms one ought to examine if, or to what extent, there may be interaction effects in the stimuli. The present analyses seek to demonstrate at least one way how this may be done.

\section{METHODS}

Interference was explored based on computer simulations carried out using Mathematica (Wolfram Research). The specifics of the simulations are explained in connection with the corresponding results.

\section{RESULTS}

A discrete Fourier Transform transforms a signal into a series of sine functions each with a different frequency. Each of these sine functions is determined by its amplitude and phase and may be represented by a vector in the complex plane in which the length represents the amplitude and the angle gives the phase (i.e., phase angle). This makes the addition of two Fourier spectra into a matter of adding two series of vectors. Such addition is illustrated in Fig. 3. In Fig. 3A are shown three sine functions of equal amplitude but with different phase (the phase values are $0, \pi / 4$, and $\pi / 2$ ). In Fig. 3B are given the vector representations of the three functions. The vectors have equal length, corresponding to the fact that they have equal amplitude, 
but differ in direction, reflecting the phase angles of the functions. Addition of the function with phase $\pi / 4$ and the one with phase $\pi / 2$ is shown in Fig. 3C. The vector representation of this addition is shown in Fig. 3D. As can be seen, the length of the combined vector for $(\pi / 4+\pi / 2)$ is shorter than the length of the vector for $\pi / 4$ plus the length of the vector for $\pi / 2$.

Given that the lengths of the vectors represent amplitudes we have that the amplitude of the sum of two sine functions is smaller than the sum of their individual amplitudes when the phases of the two functions differ. If we take one vector from the spectrum of a target stimulus and another from the spectrum of a masking stimulus (vectors representing sine functions of the same frequency) and designate them as $\mathbf{t}$ and $\mathbf{m}$, respectively, we then have that $|\mathbf{t}+\mathbf{m}|<|\mathbf{t}|+|\mathbf{m}|$ whenever the phase angle of $\mathbf{t}$ is different from that of $\mathbf{m}$. (This is known as the "triangle inequality".) This notation is based on the fact that for a complex number $z=x+y i$, with $i=\sqrt{-1}$, the absolute, i.e. amplitude or modulus, is $|z|=\sqrt{x^{2}+y^{2}}$, which corresponds to length in $\mathbf{R}^{2}$. The difference between $|\mathbf{t}+\mathbf{m}|$ and $|\mathbf{t}|+|\mathbf{m}|$ represents interference.
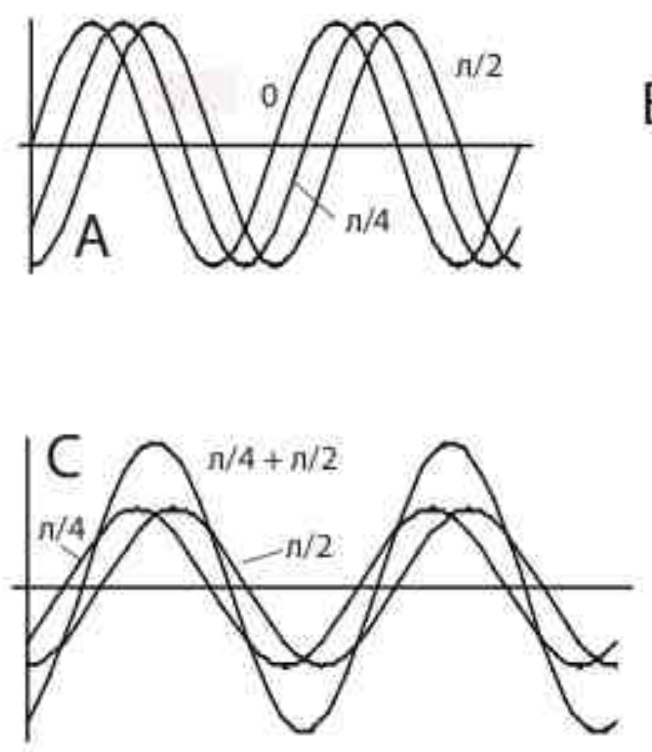
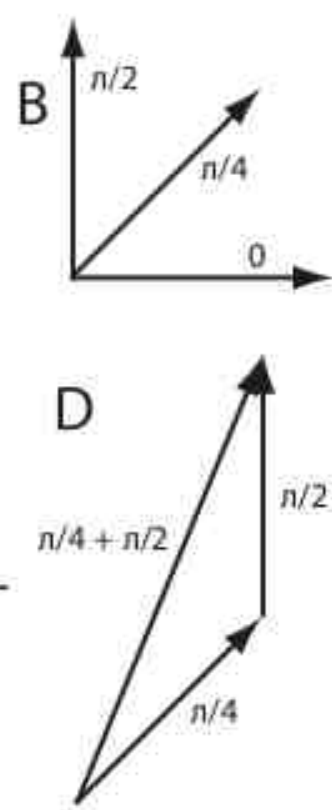

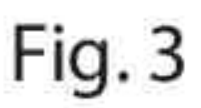

Fig. 3. (A) Three different sine functions with phases of $0, \pi / 4$, and $\pi / 2$, respectively. That is, the three functions are the same sine function with different phase angles, giving $\sin (t-0), \sin (t-\pi / 4)$, and $\sin (t-\pi / 2)$. (B) Vectors representing the three functions in A. The length of each vector represents the amplitude of the function and the angle gives the phase (i.e., phase angle). Since the three functions have the same amplitude the vectors have the same length, but the difference in phase angle means they have different directions. (C) The functions $\sin (t-\pi / 4)$ and $\sin (t-\pi / 2)$ and their addition. (D) The vector representation of the addition shown in $\mathrm{C}$. As should be apparent, the length of the vector representing the combined function (i.e., $|\sin (t-\pi / 4)+\sin (t-\pi / 2)|$ ) is smaller than the sum of the lengths of the two 
functions determined individually (i.e., $|\sin (t-\pi / 4)|+|\sin (t-\pi / 2)|)$.

Since interference is the difference between $|\mathbf{t}+\mathbf{m}|$ and $|\mathbf{t}|+|\mathbf{m}|$ it can be expressed by the ratio: $|\mathbf{t}+\mathbf{m}|$ $/(|\mathbf{t}|+|\mathbf{m}|)$. This applies to the adding of one component in the Fourier spectrum of one stimulus to the corresponding component in the spectrum of another. However, a visual stimulus, most commonly, contains many Fourier components. Thus, the amplitude of any single component cannot be used as the measure of stimulus power. Instead, as measure of stimulus power is here taken the sum of amplitudes in a given stimulus. In order to obtain a single measure of interference resulting from the adding of two stimuli the amplitudes for all components in each of the three spectra were summed giving $\sum|\mathbf{t}+\mathbf{m}|, \sum|\mathbf{t}|$ and $\sum|\mathbf{m}|$ from which the ratio $\sum|\mathbf{t}+\mathbf{m}| /\left(\sum|\mathbf{t}|+\sum|\mathbf{m}|\right)$ was computed (where $\mathbf{t}, \mathbf{m}$, and $\mathbf{t}+\mathbf{m}$ denote the Fourier spectra of the target, the masking stimulus, and the two stimuli combined, respectively). This ratio is referred to as the Relative Amplitude Sum (Skottun, 2017a, 2018a, 2018b). The amount of interference is then expressed by the amount by which the Relative Amplitude Sum falls below 1.0.

Given that one aspect of visual masking that has been studied extensively is the relative timing of target and masking stimuli the present study focuses on the effect of Stimulus Onset Asynchrony (SOA) upon the Relative Amplitude Sum. For the sake of simplicity and in order to demonstrate the basic principle of the present approach this was initially explored with 1-D stimuli. It is here being assumed that the stimulus is the deviation from the background luminance which therefore can be set to zero. Bright target and masking stimuli were represented as increments and were given the value of 1 and dark masking stimuli were represented as decrements and were given the value -1. (For a discussion of the mean luminance level see Skottun, 2018a.) Stimuli of these kinds are illustrated in Fig. 4A and Fig. 4B for light and dark masking stimuli (dashed lines), respectively. The target stimuli (solid lines) were increments in both cases. The Relative Amplitude Sum as a function of Stimulus Onset Asynchrony for the case where both the target and mask were brief luminance increments are shown in Fig. $4 \mathrm{C}$ and results for the case where the target was an increment and the mask a decrement are shown in Fig. 4D.

The plots in Fig. 4C and 4D show that the Relative Amplitude Sum is well below 1.0 when SOA $\neq 0$. According to these plots the presence of interference reduces the Relative Amplitude Sum to about 0.64. This amounts to a $36 \%$ reduction in stimulus power. 


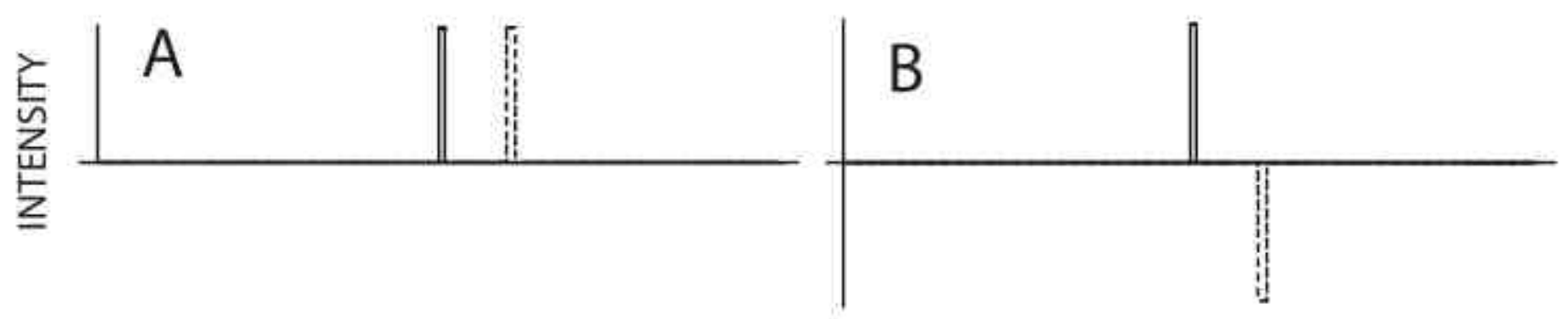

TIME

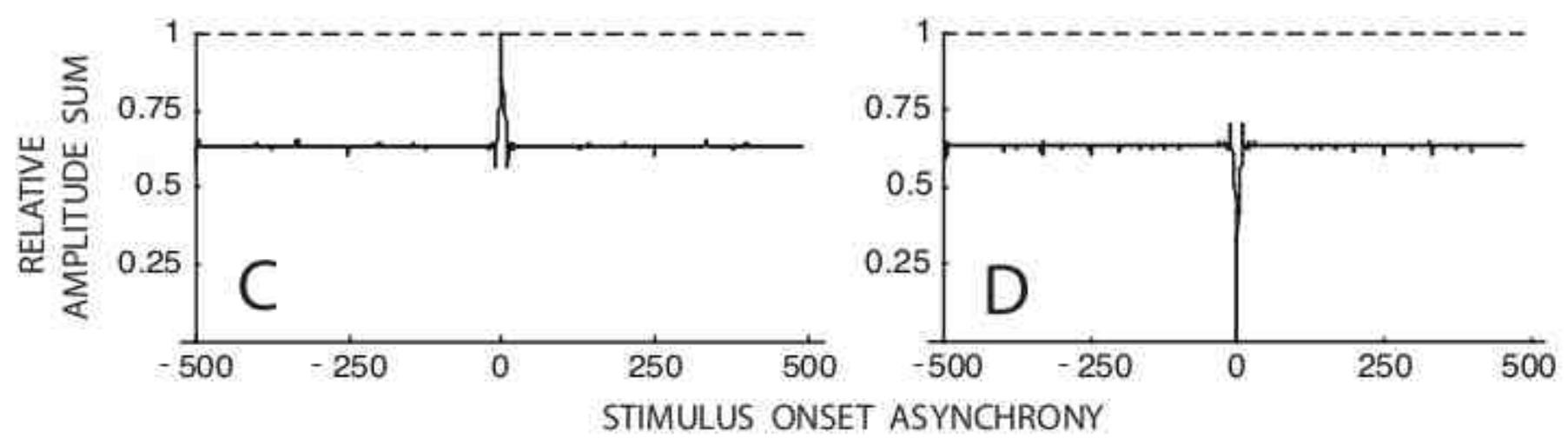

Fig. 4

Fig. 4. (A) The temporal profile of a target stimulus (solid line) and a masking stimulus (dashed line) in which both stimuli consist of brief increments of light on a dark background. (B) The same as in A but with a masking stimulus consisting of a brief reduction in luminance (dashed line). (C) Relative Amplitude Sum as a function of SOA for the stimulus constellation shown in A. (D) Same as in C but for the stimuli in B. The dashed horizontal line at 1.0 gives the Relative Amplitude Sum for when there is no interference. Interference is indicated by values below this line.

The results in Fig. 4 show not only that different stimulus elements may interfere but also that such interference may occur between elements which are not overlapping in time. In fact, in the case where the stimuli are both increments (Fig. 4A) the interference effect is absent when the two stimuli overlap completely (Fig. 4C). However, apart from the specific case of $S O A=0$ the interference effect is largely independent of the temporal relationship between the two stimuli. That is to say, provided $S O A \neq 0$ the reduction is approximately $36 \%$ irrespective of the SOA. This is clearly unrealistic since interaction effects in the visual system could only occur over limited time intervals. For instance, full temporal summation, -Bloch's Law-can only occur up to about $100 \mathrm{~ms}$ (Hart, 1992). Also, partial temporal summation, although it may extend up to as much as $1000 \mathrm{~ms}$, is limited (Legge, 1978). Thus, one would expect interference effects 


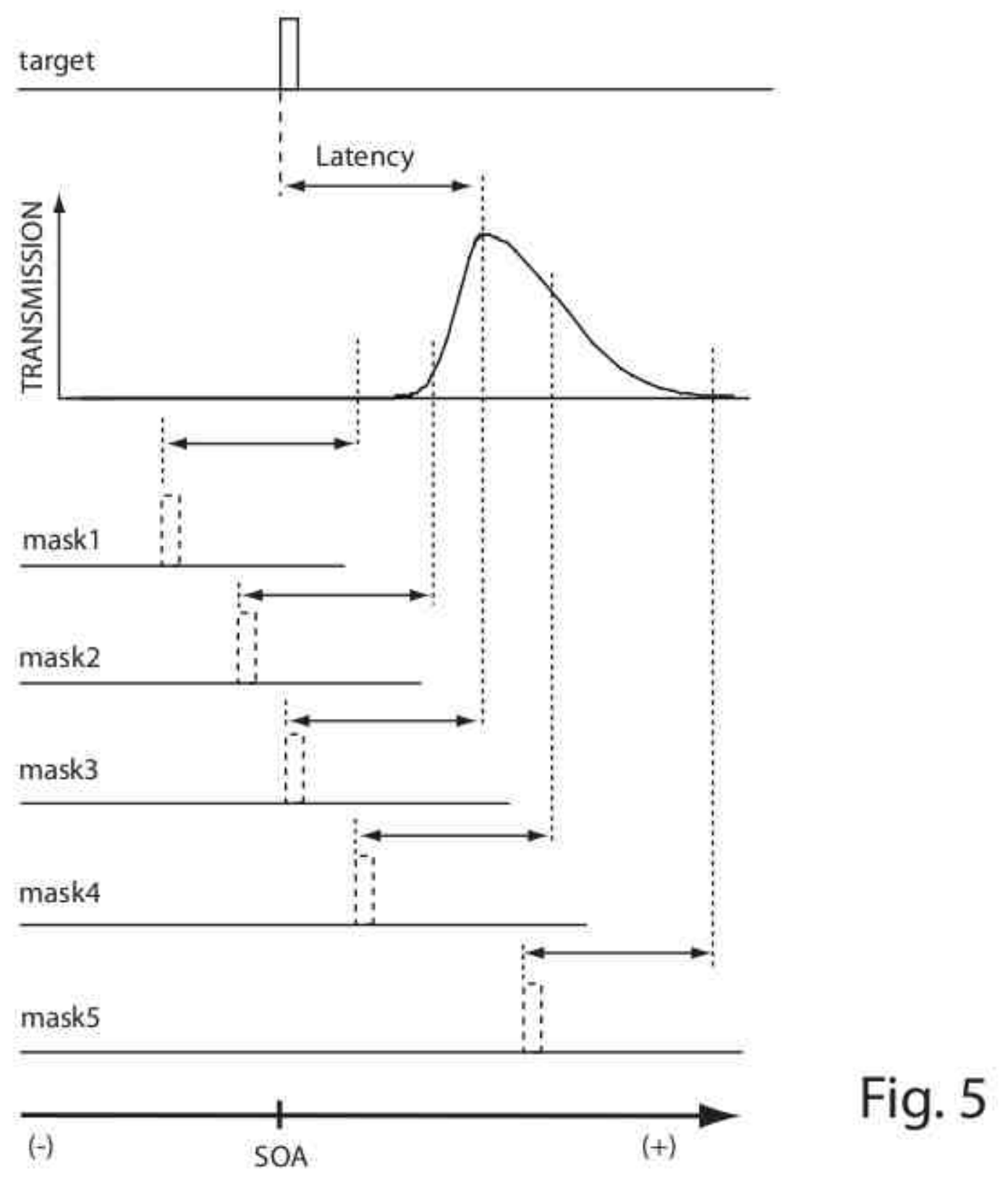

Fig. 5. The temporal integration window as a function of time relative to the presentation of the target stimulus. The upper trace shows the time profile of the target. It is assumed that this stimulus gives rise to a sensitivity function with a maximum value at some latency. It is further assumed that the masking stimuli are represented in the visual system with the same latency as the target stimulus. The lower five traces show masking stimuli with five different SOAs ranging from a masking stimulus presented well ahead of the target (mask1) to a masking stimulus presented well after the target (mask5). The vertical dotted lines mark the time of peak response to the masking stimuli.

to only take place between stimuli that are presented in somewhat close temporal proximity. In order to incorporate this a windowing function, $w\left(t-t_{0}\right)$ where $t_{0}$ denotes the time of the peak of the window, was 
introduced. This function is illustrated in Fig. 5.

Since a filter cannot be non-causal the integration window is likely to be asymmetric. It is well known that neuronal responses to abrupt stimulation tend to have a short rise-time and a shallower decay. Assuming that the integration window is linked to the neuronal response one would expect the two to have similar profiles. The present profile was generated by two Gaussians, one on the rising side and the other on the decay side, with the former having a $\sigma=1 / 3$ that of the latter. This function serves to scale the masking stimulus as a function of SOA. To do so the stimuli were multiplied with this function. (It should be noted that the profile shown in Fig. 5 is roughly similar to the hypothetical response profile for a target stimulus shown in Fig. 5 of Breitmeyer \& Ogmen, 2000 which was invoked in an attempt to account for metacontrast masking.) Further, it is assumed that the responses to the target and masking stimuli have the same latency. Thus, even though the integration takes place in the visual system at some amount of latency the relative timing of target and masking stimuli can be expressed by the SOA. (The temporal windowing function is analogous to the 2-D Gaussian window in the spatial case. See Figs. 3b and 3d of Skottun, 2017a and Fig. 6 of Skottun, 2018b.)

Since the task of a subject in a masking experiment is linked to some aspect the target stimulus the temporal integration window was located in time so as to be centered upon the onset of the target. That is to say, it had its largest transmission/sensitivity at the time of the target presentation. The interference effect following the imposition of the temporal integration window is shown in Fig. 6A and 6B. Figure 6A shows results for the case where target and mask both are increments and Fig. 6B gives results for the case where the target is an increment and the masking stimulus is a decrement. As were to be expected, the resulting curves are windowed (multiplied/scaled) versions of the functions in Fig. 4C and 4D. That is, the interference effect conforms to the shape of the temporal integration window (i.e., to an inverted version of the function in Fig. 5). 

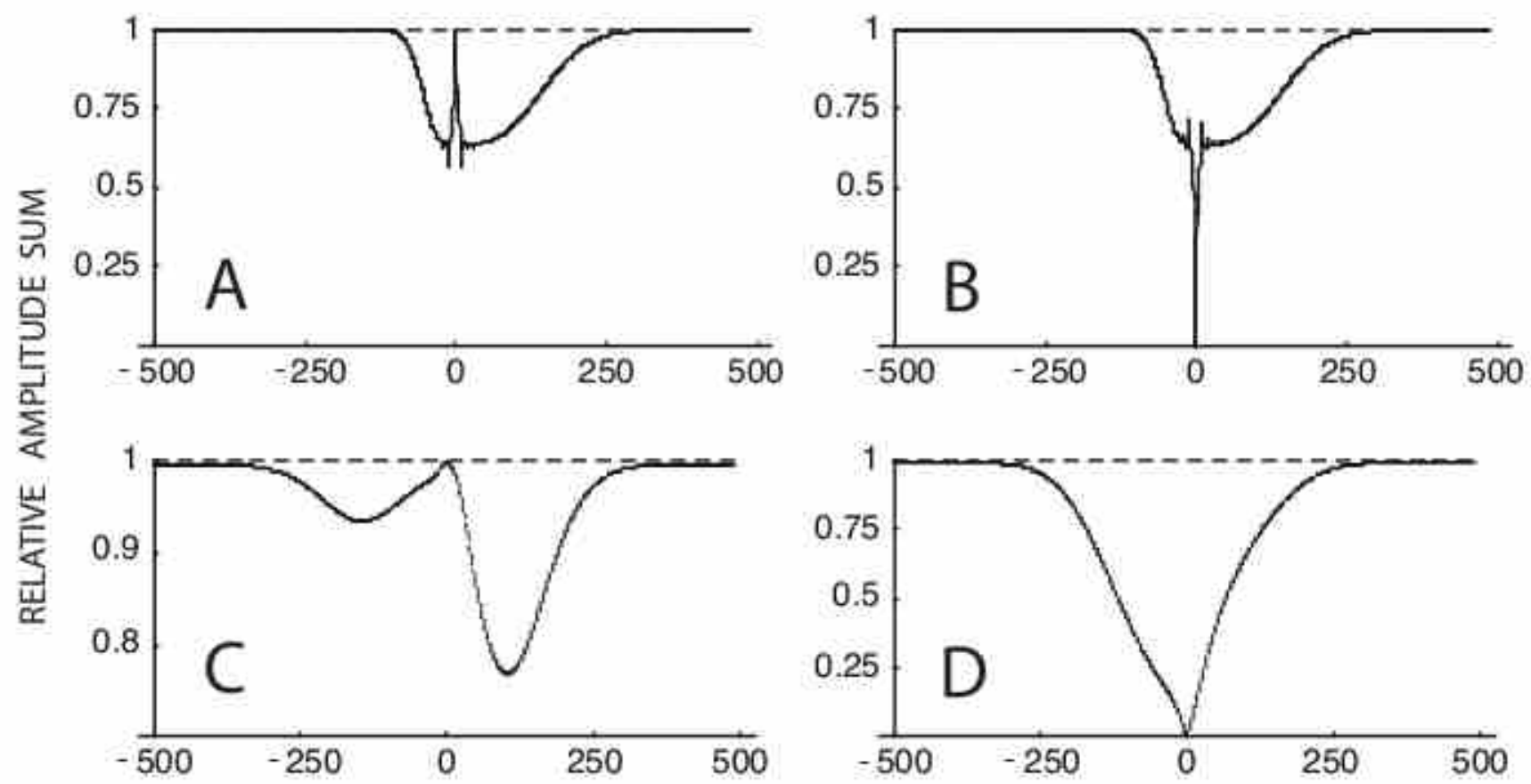

STIMULUS ONSET ASYNCHRONY

Fig. 6

Fig. 6. (A \& B) Relative Amplitude Sum when the temporal extent of the interaction has been limited by weighing (i.e., by multiplying) the stimuli with the temporal window shown in Fig. 5. The purpose of the temporal window is to limit the temporal extent of the interactions. (C \& D) Relative Amplitude Sum for when the stimuli were convolved with the time window in order to simulate temporal inaccuracy prior to the application of the time window. That is, the stimuli were first convolved with the time window and then multiplied with it. Panels $\mathrm{A}$ and $\mathrm{C}$ give results for when the target and mask both were increments and panels B and D give results for when the target was an increment and the mask a decrement.

The analyses up to this point have assumed that the stimuli are represented in the visual system with absolute accuracy. This does not seem realistic for a number of reasons. For one, neurons respond in accordance with the frequency of incoming action potentials. In order to determine frequency it is required that impulses are counted/integrated over some finite amount of time. This effect could be enhanced in a chain of several synapses. Response-time functions are added by convolution: $r_{1+2}(t)=r_{1}(t) * r_{2}(t)$ (Luce, 1986). Thus, we get for the response after $n$ synapses: $r_{(1+2 \ldots+n)}(t)=r_{1}(t) * r_{2}(t) * \ldots * r_{n}(t)$, where $r_{i}(t)$ denotes the response function at the $i$ th synapse. Unless the functions, $r_{i}(t)$, are all delta functions the chained convolutions will not only cause the response to be delayed at each synapse but also to be dilated/broadened in time. As a consequence, after a series of synapses the temporal inaccuracy may potentially be considerable. 
Also, any system which shows tuning for temporal frequency will not be able to represent events in time without some degree of inaccuracy. That the visual system shows tuning with regard to temporal frequency is well established (e.g., Kelly, 1961). As a consequence the impulse function has to cover some not insignificant amount of time (Roufs \& Blommaert, 1981; To et al., 2015). In addition, it has been shown that two stimuli need to be separated by a certain temporal interval in order to be seen as two stimuli rather than one extended one (Reeves, 1995). Thus, it is to be expected that events are represented in the visual system with degraded temporal accuracy. It appears plausible that at least part of this inaccuracy can be traced to early stages of processing. (That there should be inaccuracy in regard to time and accurate retention of temporal phase may seem contradictory. However, data indicate that both take place.)

In order to incorporate temporal inaccuracy into the model the stimuli were convolved with a temporal filter prior to the application of the temporal integration window. In the absence of more detailed information the temporal integration window was also employed as the convolution kernel. Convolution was implemented by multiplying the Fourier spectra. In more detail: (1) The Fourier Transform of the temporal filter (the convolution kernel) was multiplied with the Fourier Transform of the stimulus, (2) the Inverse Fourier Transform of the product was computed, (3) the resulting time-domain signal was then multiplied with the temporal window function, (4) the Fourier transform of the windowed signal was calculated, and (5) the Relative Amplitude Sum was calculated. In other words, a Short-Time Fourier Transform was carried out on a convolved signal. The convolution, it should be pointed out, applies to all parts of the signal irrespective of SOA and does not serve as a temporal window. Thus, both convolution and windowing are required in order to incorporate both temporal inaccuracy and temporal integration limited in time. The results of this procedure are shown in Figs. 6C and 6D. As ought to be apparent, in the case where both stimuli were increments this results in a curve showing the characteristics of Type-B masking (Fig. 6C). In the case where the target is an increments and the masking stimulus is a decrement the function shows the characteristics of Type-A masking (Fig. 6D).

The simulations using 1-D stimuli (i.e., Figs. 4 \& 6) are meant primarily to illustrate the underlying principles. It may be difficult to carry out actual experiments of these kinds. In the case of identical masking and target stimuli a subject may easily be confused as to which is which. In the case of stimuli of opposite polarity when these are presented at the same time they will cancel resulting in no stimulation. This would not be what one would call a masking paradigm. There may also take place rectification in the visual system causing an inverted signal to become equivalent to the un-inverted one. (For instance, complex cells may act 

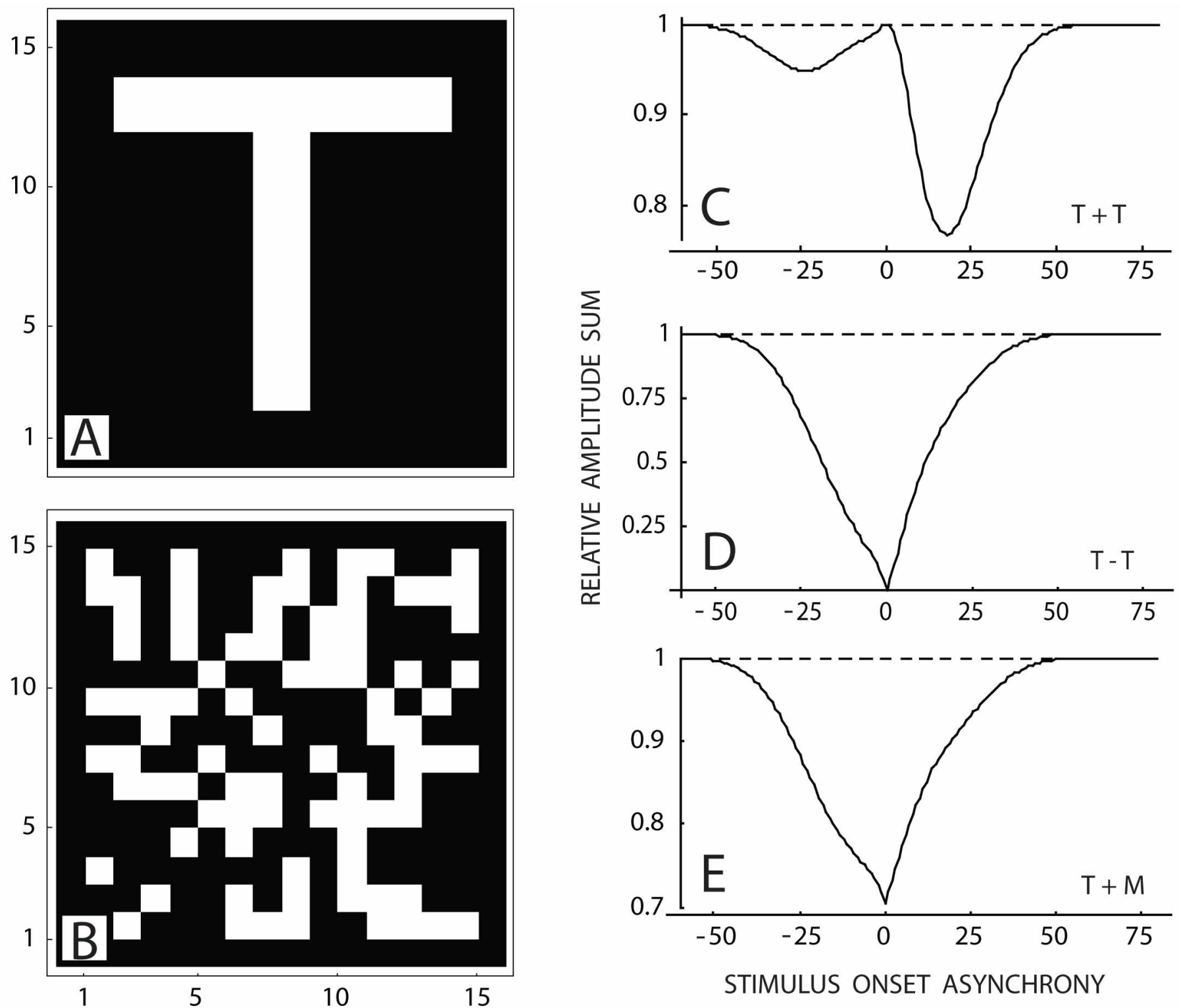

Fig. 7

Fig. 7. Interference between 2-D stimuli. (A) A 2-D target stimulus and (B) a 2-D masking stimulus. (C) Relative Amplitude Sum as a function of SOA for when the target stimulus (shown in Panel A) served as both target and masking stimuli (as indicated by "T $+\mathrm{T}$ "). (D) Relative Amplitude Sum as a function of SOA for when the target stimulus (in A) served as the target stimulus and its inverse as the masking stimulus (as indicated by "T - T"). (E) Relative Amplitude Sum as a function of SOA for when the stimulus in A was used as target and the masking stimulus shown in B was employed as the masking stimulus (as indicated by "T - M"). 
as rectifiers and a combination of outputs from ON-center and OFF-center cells may together give a rectified response.) In order to test the model with more realistic stimuli simulations were carried out with stimuli which varied along two spatial dimensions ( $\mathrm{X}$ and $\mathrm{Y}$ ) in addition to the time dimension.

A target and a masking stimulus with two spatial dimensions are shown in Figs. 7A and 7B, respectively. Each stimulus had spatial dimensions of $16 \times 16$ elements. The full stimuli were generated as 3 -D arrays with a time dimension of 140 elements giving the array the dimensions of 140, 16, and 16 elements, corresponding to time, X- and Y-dimensions, respectively. The target stimulus was inserted into the array at $t=60$ which made this $S O A=0$. This position of the target remained fixed for all tests. The mask was inserted into the array at a $t$-value that was varied systematically in order to explore the effect of SOA. (In the case where the mask was inserted at the same time as the target the two were added.) As in the cases shown in Figs. 6C and $6 \mathrm{D}$ both temporal filtering and temporal integration were applied. As shown in Fig. 5, the windowing function consisted of two Gaussians, one on the leading side and the other on the trailing side. The former had $\sigma=5$ elements and the latter $\sigma=15$ elements. Temporal filtering to simulate temporal inaccuracy was achieved by convolving the 3-D stimulus structure with the windowing function along the time dimension prior to imposing the windowing function as an integration window. (In other words, the procedure was the same as the one used to generate the results in Figs. 6C and 6D.)

Results are shown in panels C, D, and E of Fig. 7. Panel C gives results for when the target (stimulus shown in Fig. 7A) was used as both target and masking stimulus (indicated by "T + T"), and Panel D shows results for when the inverse of the target was employed as the mask (indicated by "T - T"). Panel E shows results for when the masking stimulus in Panel B served as the masking stimulus (indicated by " $\mathrm{T}$ $+\mathrm{M}$ "). Only interference along the time dimension was taken into account making these data essentially replications of the 1-D simulations shown above (i.e., Fig. 7C is essentially a replication of Fig. 6C and Fig. $7 \mathrm{D}$ is for all practical purposes a replication of Fig. 6D). These data confirm, therefore, that the results obtained with 2-D spatial stimuli (i.e., 3-D space-time stimuli) are consistent with those obtained with 1 -D stimuli. 

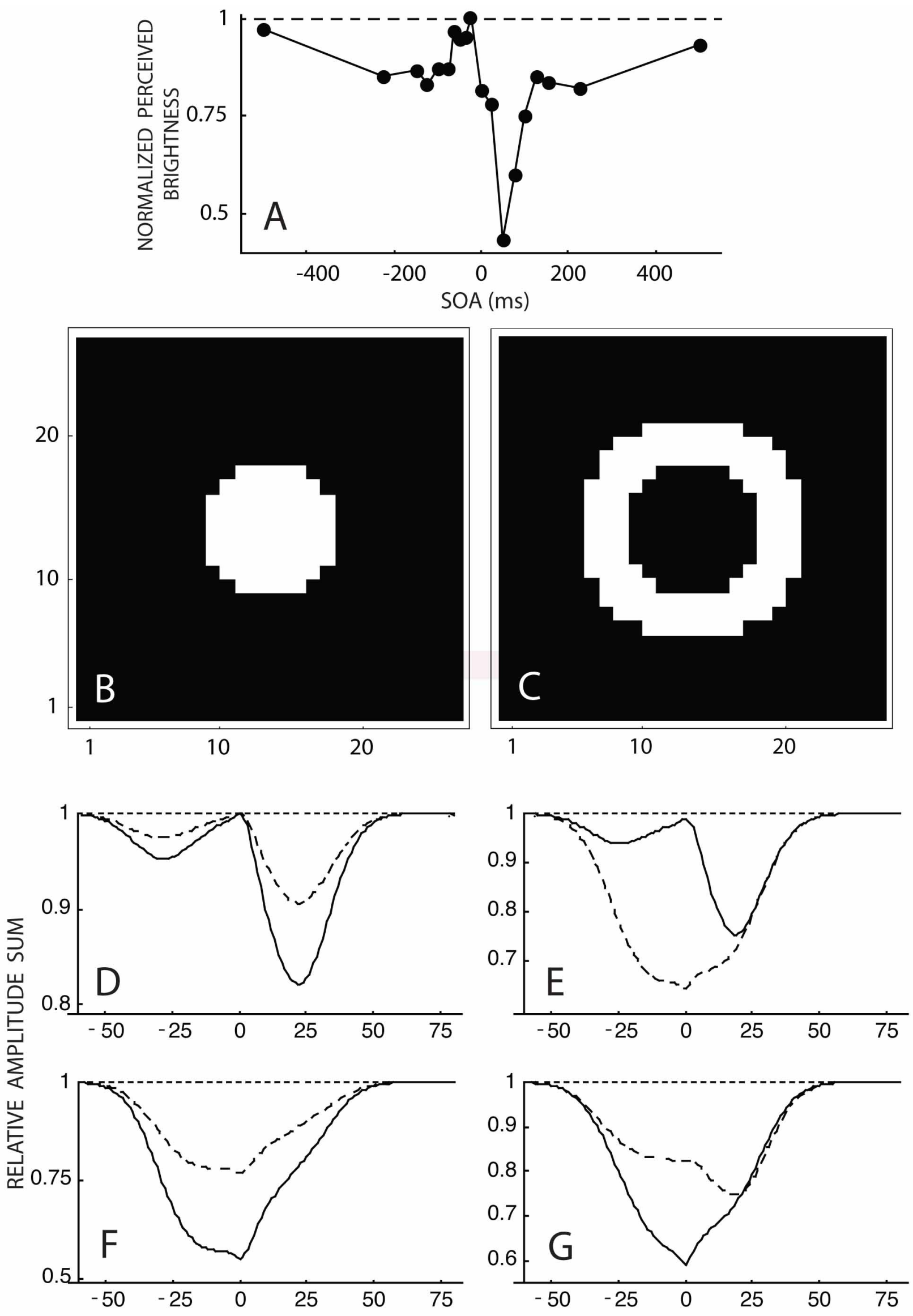

STIMULUS 15 NSET ASYNCHRONY

Fig. 8 
Fig. 8. (A) Example of a metacontrast masking function re-plotted from Fig. 6 of Ogmen et al. (2003). (B) The target stimulus used in the simulations of metacontrast. The target was a light disk with radius of 5 elements. (C) The masking stimulus was an annulus with inner radius of 5 elements and outer radius of 8 elements. (The two stimuli abutted but did not overlap.) (D) Relative Amplitude Sum as a function of SOA for the case where only interference along the time dimension was taken into account. Data are shown for two amounts of spatial blurring: Convolution with a 2-D spatial Gaussian having a $\sigma=1.0$ (dashed line) and $\sigma=2.0$ (solid line). (E) Relative Amplitude Sum as a function of SOA for the case where both spatial and temporal interference were taken into account. Data are shown for convolution with 2-D spatial Gaussians having $\sigma=2.0$ (dashed line) and $\sigma=6$ elements (solid line). Horizontal dotted line at the Relative Amplitude Sum of 1.0 in D and E represents the case where no spatial blurring (i.e., no spatial convolution) was imposed. The results in panels $\mathrm{D}$ and $\mathrm{E}$ were obtained with both the target and the masking stimuli being increments. (F \& G) Relative Amplitude Sum as a function of SOA for when the target was an increment and the masking stimulus was a decrement. Panel F gives results for where only temporal interference was taken into account whereas Panel G gives results for when interference along the time dimension as well as along the two spatial dimensions were taken into account. Dashed and solid lines in $\mathrm{F}$ give results for convolutions with Gaussians having $\sigma=1.0$ and $\sigma=2.0$, respectively. Results in $\mathrm{G}$ are given for when there was no spatial convolution (dashed line) and for when a Gaussian with $\sigma=2$ was used (solid line). The dotted horizontal lines at 1.0 in panels $\mathrm{E}$ and $\mathrm{G}$ mark no interference.

Type-B masking has commonly been obtained with metacontrast masking stimuli. These are stimuli in which target and mask occupy different and non-overlapping regions of the visual field. Typically the mask is shaped so as to surround the target. In Fig. 8A is shown metacontrast data re-plotted from Fig. 6 of Ogmen et al. (2003). As can be seen, the masking function in this plot resembles the interference functions shown in Fig. 6C and Fig. 7C. This prompts the question of whether or not the present simulations are able to also generate interference functions similar to Type-B masking functions from metacontrast stimuli.

Metacontrast stimuli were generated by having a circular disk of 5 elements radius be the target and an annular ring with inner radius of 5 elements and outer radius of 8 elements be the masking stimulus. The stimuli were generated in $27 \times 27$ element arrays and are illustrated in Fig. 8B and 8C. The analyses were the same as those used for generating the data in Fig. $7 \mathrm{C}, 7 \mathrm{D}$, and $7 \mathrm{E}$ with the exception that the 3-D stimulus array had the dimensions $140 \times 27 \times 27$ (corresponding to time, X-, and Y-dimensions).

Relative Amplitude Sum as a function of SOA is shown in Figs. 8D, 8E, 8F, and 8G. Results for the case where both stimuli are increments and only interference along the time dimension is considered are shown in Fig. 8D. In this case there is no interference as indicated by the horizontal dotted line at the Relative Amplitude Sum of 1.0. This is precisely what is to be expected since the target and masking stimuli are non-overlapping spatially. If, on the other hand, spatial inaccuracy is introduced the situation changes. Such inaccuracy was simulated by convolving the spatial stimuli with a 2-D Gaussian. The results of this operation are shown for convolution with 2-D spatial Gaussians having $\sigma=1$ (dashed line) and $\sigma=2$ (solid line). As can be seen, adding spatial inaccuracy introduces interference. In both cases (i.e., for both $\sigma=1$ 
and $\sigma=2$ ) the curves show the characteristics of Type-B masking.

Allowing interference to take place along both the time dimension and the two spatial dimensions alters the results [FOOTNOTE 1]. This is shown in Fig. 8E. In this case when the stimuli are spatially convolved with a spatial Gaussian with $\sigma=2$ elements the interference effect takes on the shape of Type-A masking (dashed line). On the other hand when the $\sigma$ of the spatial filter is increased to 6 elements a function showing the characteristics of Type-B masking emerges (solid line).

It has been reported that Type-B masking curves may be obtained from metacontrast stimuli when the mask and target stimuli are of opposite polarity (Breitmeyer et al., 2008. But see Becker \& Anstis, 2004). In order to explore such stimulus conditions the test was repeated with a negative masking stimulus (i.e., the target was an increment and the masking stimulus a decrement). The results of this analysis are shown in Figs. $8 \mathrm{~F}$ and $8 \mathrm{G}$, where Fig. $8 \mathrm{~F}$ gives results for interference only along the time dimension whereas Fig. $8 \mathrm{G}$ shows results for when interference along all three dimensions are included. As can be seen, the interference effects tends to take on the appearance of Type-A masking. On the other hand, the case of interference along all three dimensions (Fig. 8G) there is some evidence for a pattern resembling Type-B masking when there is no spatial convolution (dashed line in Fig. 8G). These analyses do not take account of any potential rectification in the visual system. However, as was pointed out above, it is not clear how polarity reversals are mediated by the visual system. Thus, the relevance of reversals in stimulus polarity is unclear.

Enns and Di Lollo (1997) described what they termed "Masking by Continuation". In this kind of masking, as in metacontrast, the masking stimuli do not overlap with the target, However, unlike the masking stimulus in metacontrast where the masking stimulus typically consists of a continuous contour surrounding the target the masking stimulus used by Enns and Di Lollo to generate Masking by Continuation consisted of four small squares. Stimuli modeled after those of Enns and Di Lollo are shown in Fig. 9A. The stimuli were generated as $64 \times 64$ elements array. In Fig. 9A the target and the masking stimuli are shown together. The target is the black diamond which has a maximum width of 32 elements and a maximum height of 32 elements. The masking stimulus was made up of the 4 squares each 5 elements on the side. The separation between the closest corner of each of the squares and the closest edge of the diamonds was $\sqrt{5^{2}+5^{2}} \approx 7$ elements. (For estimates of interference based on the spatial aspects of such stimuli see Skottun, 2017b.)

Results re-plotted from Fig. 3D of Enns and Di Lollo (1997) are shown in Fig. 9B and the results from the present simulations are shown in Fig. 9C. These data incorporate interference effects along both the two spatial dimensions and the time dimension (i.e., the procedure used was the same as the one used to generate 
the results in Figs. 8E and 8G). Results are shown for three levels of spatial inaccuracy: Convolution with 2-D spatial Gaussians having $\sigma=0,2$, and 6 elements. As can be seen, the largest masking effect is found when the masking stimulus is presented after the target. This is particularly pronounced when some degree of spatial inaccuracy is incorporated (i.e., for data obtained with $\sigma=2$ and 6 shown with curves marked "2" and "6"). These results demonstrate that in the case of stimuli used to generate Masking by Continuation it is possible to obtain interference functions which are not symmetric around SOA $=0$ [FOOTNOTE 2].

It is interesting to note the similarity between the results in Fig. 9C and those in Fig. 8E, i.e. between results obtained with metacontrast stimuli and those obtained with Masking by Continuation stimuli, given that Enns and Di Lollo (2017, p. 137) in connection with stimuli presented parafoveally wrote: "However, the most striking result was the strong resemblance between four-dot and metacontrast masking".

\section{DISCUSSION}

From the present analyses it ought to be quite clear that when combining two stimuli the sum of amplitudes in the combined stimulus may be smaller than the sum of the amplitudes in the two initial stimuli determined separately (i.e., $\sum|\mathbf{t}+\mathbf{m}|$ may be smaller than $\sum|\mathbf{t}|+\sum|\mathbf{m}|$ ). Assuming that the sums of amplitudes is a measure of stimulus power the present results entail that the stimulus power of the combined stimulus may be smaller than the stimulus power of the two component stimuli added. This has been demonstrated previously in the case of spatial interactions (Skottun, 2017a, 2017b, 2018). The present analyses have sought to gain insight into this issue under the kinds of conditions found in relation to visual masking.

The present observations illustrate how interference may be present under the kinds of conditions found in connection with visual masking. However, the interference effects described here are for the combined target-and-masking stimuli. When $\sum|\mathbf{t}+\mathbf{m}|<\left(\sum|\mathbf{t}|+\sum|\mathbf{m}|\right)$ this entails that the full amplitudes in the target and the masking stimulus cannot both be contained in the amplitudes of the combined stimulus. This means that either the amplitudes in the target, in the masking stimulus, or in both stimuli are reduced when the stimuli are combined. That only the target or the masking stimulus should be affected would be extremely unlikely. What is of particular importance in relation to masking is whether or not the stimulus power of the target is reduced. The present results do not allow us to address this specifically. But, were we to assume that the target and masking stimuli were equally affected by interference the present results would represent valid estimates of the effects of interference upon the target stimuli. 

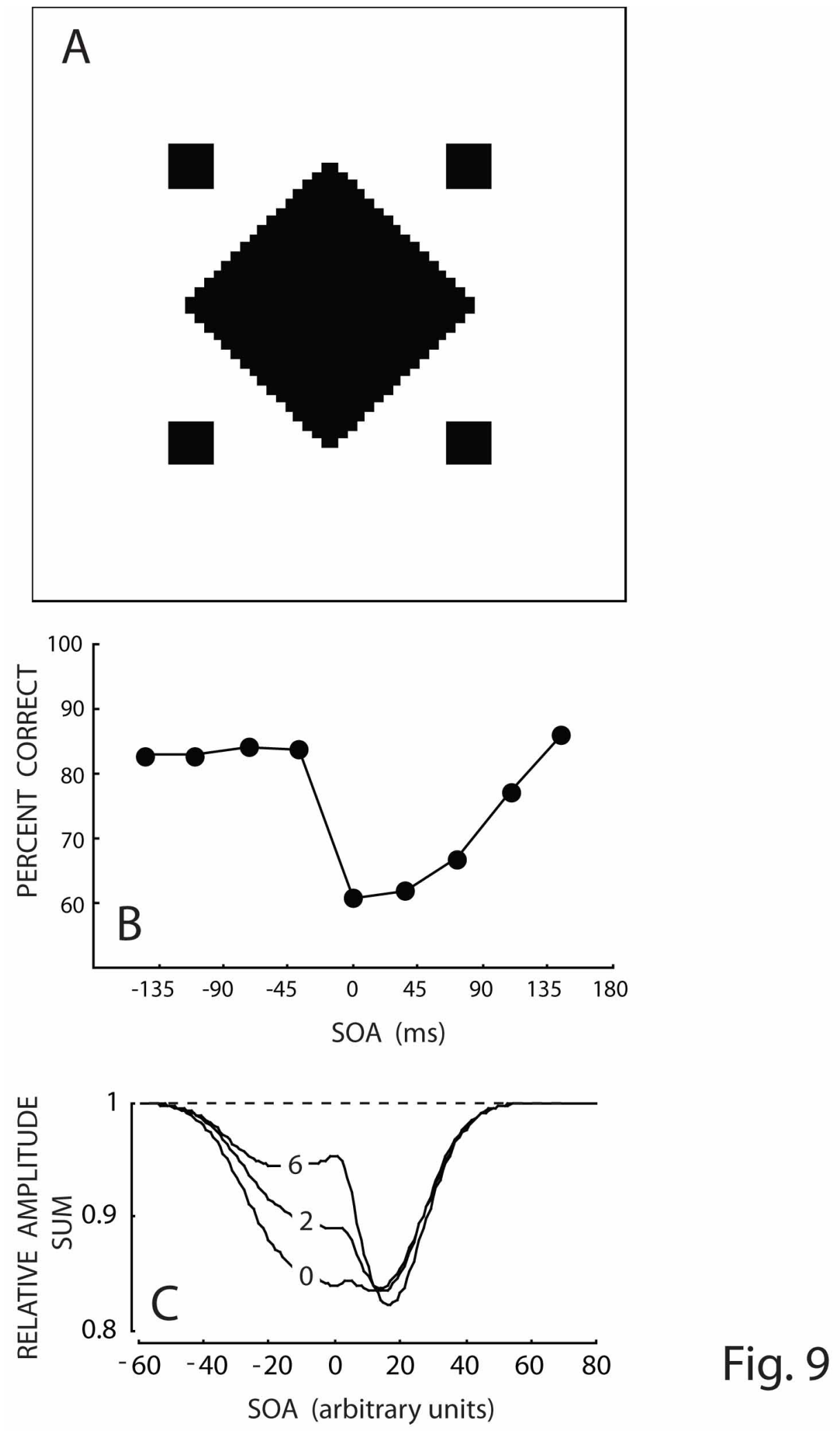

Fig. 9. (A) Stimuli employed to simulate Masking by Continuation. The target and masking stimuli are shown together. The target was the central diamond and the masking stimulus consisted of the four squares. The stimuli had the dimensions of $64 \times 64$ elements. (B) Data re-plotted from Fig. 3D of Enns and Di Lollo (1997). (C) Relative Amplitude Sum as a function of SOA for the stimuli shown in Panel A. Results are 
shown for three levels of spatial inaccuracy: No spatial convolution (marked "0"), convolution by 2-D spatial Gaussian with $\sigma=2$ (curve marked "2") and convolution with a Gaussian with $\sigma=6$ (curve marked "6").

Although the analyses have incorporated aspects of the visual system (i.e., temporal inaccuracy incorporated by convolution and temporal integration) the interference effect is between elements in the stimuli or between their representations. Interference is a basic aspect of the combining of signals. In other words, there is no special neural or neuronal "mechanism" generating interference.

It may come as a surprise that there may be interference between two stimuli which do not overlap in time. However, under some conditions it may be precisely when the stimuli are not overlapping the interference is the most pronounced. The reason for this would be that the time difference causes Fourier components in the spectra of the two stimuli to differ in temporal phase. As was pointed out in connection with Fig. 3 interference effects are the result of phase differences.

The present analyses are based on the amplitudes of sine functions. There are a number of reasons for basing analyses on such functions. For instance Campbell and Robson (1968) found that complex stimuli are detected when one of the Fourier components in the stimuli reaches threshold. Also, that neurons in the primary visual cortex (cortical Area V1) respond to sine gratings is well established (De Valois et al., 1982). It has been demonstrated that cortical cells respond to checkerboard stimuli in accordance with the amplitudes in the Fourier spectra rather than the edges in the stimuli (De Valois et al., 1979). Also, the directional selectivity of cortical cells to drifting patterns of random dots can be accounted for on the basis of the spatio-temporal amplitude spectra of the stimuli (Skottun et al., 1994). Observations of these kinds suggest that sine functions are of importance. This has led to the proposal that the visual system carries out a localized Fourier Transform (De Valois \& De Valois, 1990).

That amplitudes are important seems quite clear given that the responses of visual neurons, to a large extend, depend upon the amplitudes (i.e., contrast) of the stimuli. This applies both at the subcortical and the cortical levels. Most commonly, responses increase with increasing amplitudes (i.e., increasing contrast) (Sclar et al., 1990). Thus, anything that alters the amplitudes linked to a given stimulus would be expected to be in a position to influence its visibility.

The fact that interference is here described in terms of Fourier analysis and amplitudes may create the impression that interference is a result of the Fourier Transform and that it depends upon the Fourier Transform. This, however, would represent a misunderstanding. Interference can be demonstrated based 
on the norm of the Fourier Transform (Skottun, 2017b, 2018a, 2018b). That is, the norm of the Fourier Transform of two stimuli combined can be smaller than the sum of the norms of the Transforms of the two stimuli determined separately. What makes this significant is that the norm of the Fourier Transform is equal to the norm of the space/time domain representation of the stimuli. From this it follows that if interference can be demonstrated with the norms of the Fourier Transform it can also be demonstrated with space/time domain norms . In other words, it can be demonstrated in the space/time domain. Interference can also be demonstrated based on the the sum of the absolutes of wavelet coefficients (Skottun, 2018). Thus, there are three different measures of "stimulus power" which all are susceptible to interference. In fact, if we were to require that "stimulus power" should be determined so that a stimulus added to itself has twice the stimulus power it becomes difficult to conceive of a plausible measure of stimulus power which is not (Skottun, 2018). The focus on amplitudes in the present report is due to the fact it is relatively simple to understand and explain interference in terms of the adding of sine functions (see e.g., Fig. 1 of Skottun 2017a, 2017b, 2018a, 2018b).

Some authors have used the term "interference" when describing the interactions between stimuli in visual masking experiments (e.g., Di Lollo et al., 1974; Enns, 2004) and Legge and Foley (1980, p. 1458) described "masking" as a term "... used commonly to refer to any destructive interaction or interference among transient stimuli that are closely coupled in space or time". However, in these cases the term "interference" has been used in a non-specific sense to merely mean that one stimulus influences or interacts with another or with the perception of another stimulus. In the present context the term "interference" is used in a specific sense to mean an interaction which depends on the relative temporal phase of the Fourier components of the stimuli. (In this connection it may be worth noting that some forms of auditory masking have been conceptualized in terms of interference. This is commonly referred to as "energetic masking". Mattys et al., 2012.)

In order for there to be interference between two stimuli presented at different times it is required that the two stimuli fall within the same temporal window of integration. As was pointed out above, full temporal summation may extend to $100 \mathrm{~ms}$ (Hart, 1992) and partial summation may extend to as much as $1000 \mathrm{~ms}$ (Legge, 1978). Thus, stimuli presented at somewhat different times would be expected to be able to generate interference. However, given the fact that temporal summation is limited it seems also quite clear that stimuli presented at widely different times may not interact. It was precisely to incorporate this fact that a temporal integration window (cf. Fig. 5) was incorporated into the analyses. 
The interference effect as a function of SOA may resemble either Type-A (Figs. 6D, 7D, 7E, 8E, 8F, \& 8G) or Type-B masking (Figs. 6C, 7C, 8D, 8E, 8G, \& 9C). When a skewed temporal integration window (as shown in Fig. 5) is employed it is a relatively simple matter to obtain interference functions resembling Type-B masking. A skewed window, i.e. one with a rapid increase and a more slow decline, is plausible given that the window has to be non-causal. That is to say, it cannot respond to events that have yet to occur. (In other words, there cannot be negative latencies.) It is well known that neuronal responses to abrupt stimulation have a short rise-time and a longer decay. Assuming that the integration window is linked to the neuronal response one would expect the integration window to have a similar profile.

In addition to temporal summation interference also requires that temporal phase information be retained. That phase information is retained is demonstrated by experiments exploring what is known as "Phantom Contours" which show that although absolute phase information is retained up to only about $5-7 \mathrm{~Hz}$ (Rogers-Ramachandran \& Ramachandran, 1998) information about relative phase is retained at higher temporal frequencies. The evidence for this is that if one creates a situation in which one set of elements modulates in-phase and second set of elements modulates out-of-phase with those in the first set, a boundary (the so called Phantom Contour) can be seen separating the two sets of elements at frequencies above 15 Hz (Rogers-Ramachandran \& Ramachandran, 1998). This demonstrates, therefore, that relative temporal phase information is retained even at quite high temporal frequencies. In the present context relative phase information (rather than absolute phase information) is what is relevant.

A further requirement is that the two stimuli to be combined have energy at the same components in their Fourier spectra. In regard to metacontrast it is therefore of interest to note that metacontrast masking requires that the target and mask have energy at the same orientations (Ishikawa et al., 2006). In this connection it should also be noted that the imposition of a window in the time domain may cause the Fourier spectra to widen allowing for more overlap between the frequency spectra of target and masking stimuli. Also, in order to make the stimuli be well defined in regard to time they have typically been created so as to have abrupt onset and offset. This will inevitable cause them to have relatively broad frequency spectra, which also increases the opportunity for the spectra of the target and mask to overlap. Thus, the desire to have well defined stimuli in time may make them susceptible to interference effects.

Masking presumably involves the combined effect of a number of factors. It seems therefore implausible that it could be explained by a single principle. For instance, exception for the interference itself the analyses have assumed linear processing. This may not be fully realistic given that nonlinearities in the visual system 
are ubiquitous. In addition, the functions employed in order to simulate temporal integration and temporal inaccuracy may differ somewhat from the actual functions. Furthermore, if, or to what extent, visual masking reflects interference between stimuli requires detailed comparisons between stimuli and responses under the particular conditions employed in particular experiments. One thing that ought to be quite clear from the present analyses is that even though a target stimulus remains physically the same irrespective of whether or not it is presented in combination with a masking stimulus it cannot be counted upon to have the same stimulus power under the two conditions. This may apply also when the stimuli are presented at slightly different times.

Because the analyses deal primarily with stimuli they do not represent an attempt to explain, or to provide a theory of, visual masking which is a matter of response to visual stimuli. It seems that in order to understand responses to visual stimuli one ought first try to understand the stimuli. The present analyses represents an attempt at this and an attempt at providing a framework for such an understanding. In so doing the analyses seek to provide a foundation upon which theories may be constructed.

\section{FOOTNOTES}

1. The signal along the time dimension was Fourier analyzed for each $\mathrm{x}, \mathrm{y}$ position and the interference was calculated as described based on summing the amplitudes. In the case where also spatial interference was taken into account the analyses were based on a full 3-D Fourier Transform. For information on the 3-D Fourier Transform see Skottun (2013).

2. Estimates of interference in purely spatial 2-D representation of stimuli of the kinds shown in Fig. 9A have indicated that it is necessary to set the dc value equal to zero. This was also done in these calculations. However, tests indicate that this is of minor importance in the present 3-D case. For a discussion of the dc value in relation to interference see Skottun 2018a.

\section{REFERENCES}

Breitmeyer B.G. \& Ganz, L. (1976) Implications of Sustained and Transient Channels for Theories of Visual Pattern Masking, Saccadic Suppression, and Information Processing. Psychological Review, 83, 1-36.

Breitmeyer, B.G., Kafaligonul, H. \& Ogmen, H. (2008) Metacontrast masking and stimulus contrast polarity. Visual Research, 48, 2433-2438.

Breitmeyer, B.G., \& Ogmen, H. (2000) Recent models and findings in visual backward masking: A comparison, review, and update. Perception \& Psychophysics, 62, 1572-1595. 
Campbell, F.W. \& Robson, J.G. (1968) Application of Fourier analysis to the visibility of gratings. Journal of Physiology, 197, 551-566.

De Valois, R.L., \& De Valois, K.K. (1990) Spatial Vision. Oxford University Press. New York, NY, USA.

De Valois, R.L., Albrecht, D.G., \& Thorell, L.G. (1982) Spatial frequency selectivity of cells in Macaque visual cortex. Vision Research, 22, 545-559.

De Valois, K.K., De Valois, R.L. \& Yund, E.W. (1979) Responses of striate cortex cells to gratings and checkerboard patterns. Journal of Physiology, London, 291, 483-505.

Di Lollo, V., Lowe, D.G., \& Scott, J.P. Jr. (1974) Backward masking and interference with the processing of brief visual displays. Journal of Experimental Psychology 103, 934-940.

Enns, J.T. (2004) Object substitution and its relation to other forms of visual masking. Vision Research 44, 1321-1331.

Enns, J.T., \& Di Lollo, V. (1997) Object substitution: A new form of masking in unattended visual locations. Psychological Science. 8, 135-139.

Enns, J.T., \& Di Lollo, V. (2000) Whats new in visual masking?Trends in Cognitive Sciences. 4, 345-352.

Hart, W.M. (1992) The temporal responsiveness of vision. In Adlers Physiology of the Eye. Hart, W. M. (ed.) Mosby-Year Book, Inc., St. Louis, MI. USA. pp. 548-578.

Hellige, J.B., Walsh, D.A., Lawrence, \& Prasse, M. (1979) Figure relationship effects and mechanisms of visual masking. Journal of Experimental Psychology: Human Perception and Performance, 5, 88-100.

Ishikawa, A., Shimegi, S., \& Sato, H. (2006) Metacontrast masking suggests interaction between visual pathways with different spatial and temporal properties. Vision Research, 46, 2130-2138.

Kelly, D.H. (1961) Visual responses to time-dependent stimuli. I. Amplitude sensitivity measurements. Journal of the optical Society of America. 51, 422-429.

Legge, G.E. (1978) Sustained and transient mechanisms in human vision: Temporal and spatial properties. Vision Research, 18, 69-81.

Luce R.D. (1986) Response times. Oxford University Press. New York.

Mattys, S.L., Davis, M.H., Bradlow, A.R., \& Scott, S.K. (2012) Speech recognition in adverse conditions: A review. Language and Cognitive Processes, 27, 953-978.

Ogmen, H., Breitmeyer, B.G., \& Melvin, R. (2003) The what and where in visual masking. Vision Research 43, $1337-1350$.

Reeves, A. (1995) Temporal resolution in visual perception. In Handbook of Perception and Action. 1. Perception. Prinz. W. \& Bridgeman, B. (eds.) Academic Press, London, UK. pp. 11-24.

Rogers-Ramachandran, D.C., \& Ramachandran, V.S. (1998) Psychophysical evidence for boundary and surface systems in human vision. Vision Research, 38, 71-77.

Roufs, J.A.J., \& Blommaert, F.J.J. ( 1981) Temporal impulse and step responses of the human eye obtained psychophysically by means of drift-correcting perturbation technique. Vision Research, 21, 1203-1221.

Sclar, G., Maunsell, J.H.R., \& Lennie, P. (1990) Coding of image contrast in central visual pathways of the macaque monkey. Vision Research, 30, 1-10.

Skottun, B.C. (2013) A few observations on metacontrast stimuli. Journal of Integrative Neuroscience. 12, $163-182$.

Skottun, B.C. (2017a) A few remarks on spatial interference in visual stimuli. Behavior Research Methods. In Press. DOI: 10.3758/s13428-017-0978-3

Skottun, B.C. (2017b) Interference in stimuli employed to assess masking by substitution. PsyArXiv. doi: 10.17605/OSF.IO/XM3AU. 
Skottun, B.C. (2018a) A few remarks on stimulus power and interference in visual stimuli. PsyArXiv. DOI: 10.17605/OSF.IO/3NT84.

Skottun, B. C. (2018b) Interference in lateral masking stimuli-The effects of relative phase, position, orientation, and spatial frequency. Behavioral Brain Research. In Press. DOI: 10.1016/j.bbr.2018.04.016.

Skottun, B.C., Zhang, J., \& Grosof, D.H. (1994) On the directional selectivity of cells in the visual cortex to drifting dot patterns. Visual Neuroscience, 11, 885-897.

To, M.P.S., Gilchrist, I.D., \& Tolhurst, D.J. (2015) Perception of differences in naturalistic dynamic scenes, and a V1-based model. Journal of Vision, 15(1), 19, 1-13. 